\title{
Transverse Flux Permanent Magnet Generator for Ocean Wave Energy Conversion
}

\author{
José Lima, Anabela Pronto, and Mário Ventim Neves \\ Faculdade de Ciências e Tecnologia - Universidade Nova de Lisboa, \\ Quinta da Torre, 2829-516 Caparica, Portugal \\ jose.a.o.lima@gmail.com, amglefct.unl.pt, ventim@uninova.pt
}

\begin{abstract}
Modern energy demands led the scientific community to renewable energy sources, such as ocean wave energy. The present work describes a model for a cost efficient rotary electrical generator, optimized for ocean wave energy conversion. The electrical power, supplied by low speed mechanical movement, requires the use of electrical machinery capable of generating high amounts of torque. Among the analyzed topologies, the one selected for further study was the Transverse Flux Permanent Magnet machine (TFPM). This topology differs from the conventional ones, presenting high power and torque densities, and allowing to independently set machine current and magnetic loadings in the machine. The machine was designed and analyzed through the use of a 3D FEM software. The obtained results show that the TFPM is a strong candidate to be used in large scale converting systems.
\end{abstract}

Keywords: Transverse flux, TFPM, Ocean wave energy, Low Speed, Generator, Finite elements.

\section{Introduction}

The ocean presents itself as an inexhaustible source of clean and renewable energy that appears mainly in the form of ocean waves, generated by the action of wind on the ocean surface, and in the form of ocean currents, caused by the effect of tides and by variations of salinity and temperature. Nowadays, there's a growing worldwide demand for energy resources that reveal themselves as alternatives to the existing ones, highly pollutant and with limited availability. Therefore, conditions must be created for their exploitation in a sustainable manner. Since the beginning of research on wave energy, encouraged by the oil crisis [1], several devices have been proposed to exploit this resource, although only a small number of these have been studied and implemented on a large scale [2]. Due to the complexity of ocean waves' characteristics to extract energy, the development of technologies that may take advantage of this energy source requires further research.

A vast knowledge about the physical aspects of ocean wave energy already exists [3] [4]. However, there is still no consensus on the best technology to take advantage of this resource.

The current work attempts a qualitative research on the main topologies of electrical machines that may allow a direct and efficient exploitation of low speed 
rotational movement, and the selection, sizing and optimization of a topology for a small-scale electrical generator prototype. For each topology, the various pros and cons were considered. The Transverse Flux Permanent Magnet machine with flux concentrators was selected as the object of study, regarding its favorable characteristics for ocean wave energy conversion.

\section{Contribution to Sustainability}

Sustainability of Earth's energy resources urgently demands the optimization of its use, due to the current energy requirements and consumption. This work takes a further step towards turning ocean wave energy into a viable and desirable energy source. The developed generator's characteristics may act as an incentive to the expansion of ocean wave energy conversion.

\section{Selected Electrical Generator}

\subsection{Direct Drive Approach}

The ocean's environment is variable and unstable. A system with the purpose of converting mechanical ocean wave energy into electrical energy must be prepared to generate, with relatively high quality and efficiency, energy that meets the functional requirements of the electrical grid. Given the slow and undulatory motion of ocean waves, and to avoid the use of expensive gearboxes with periodic maintenance requirements, the development of an efficient direct drive electric generator was one of the main goals of this work. Thus, it becomes necessary to employ a high torque density machine, and therefore with a high number of poles.

\subsection{Transverse Flux Permanent Magnet (TFPM) Topology}

Several electrical generator topologies were analyzed. The TFPM topology seems to have great potential for ocean wave energy conversion, showing better use of the machine size and permanent magnet materials when compared to conventional and other non-conventional topologies [5]. Besides the possibility of reaching a very high torque density, by increasing the number of poles, it allows to set the electric current density regardless of the magnetic flux, unlike conventional radial topology machines, where the cross sectional area of air gap competes directly with the windings for the available space [6] [7] [8] [9]. In TFPM the air gap area defines the current density, while the axial length defines the magnetic flux density.

Due to its characteristics, the TFPM, with flux concentrators, was the topology selected for this research work.

\section{Sizing}

As aforementioned, the main objective of this work was to develop a model for a rotating electrical generator capable of operating at low speeds, allowing the 
conversion of ocean wave mechanical energy into electrical energy to be supplied to the main grid. A single-phase TFPM prototype model capable of generating an output of $10 \mathrm{~kW}$ was designed. It was considered an average angular frequency of $150 \mathrm{rpm}$ for a gearless energy conversion system, at the shaft that drives the generator. Thus, in order to comply with the grid's frequency $(50 \mathrm{~Hz})$, the machine was designed with 20 pole pairs. Optimal values for each parameter of the generator topology were calculated and simplified machine's schemes were used to obtain expressions that reflect the generator's operation conditions.

\subsection{Magnetic Circuit}

The studied topology is illustrated in Fig. 1. The generator's rotor consists in two rows of permanent magnets and flux concentrators, and a central stainless steel separator, positioned between each row of the rotor, which acts as a "magnetic insulator" since it is made of a diamagnetic material. Each of these rows contains a set of magnets polarized in the direction of rotation; each magnet is inversely polarized with its pair in the opposite row and is also followed by a flux concentrator. The permanent magnet material chosen for this analysis was the Neodymium Iron Boron $(\mathrm{NdFeB})$ due to its known magnetic properties [10], with a remanence $B_{\mathrm{R}}=1,4 \mathrm{~T}$ and a coercive field $H_{\mathrm{C}}=795 \mathrm{kA} \cdot \mathrm{m}^{-1}$.

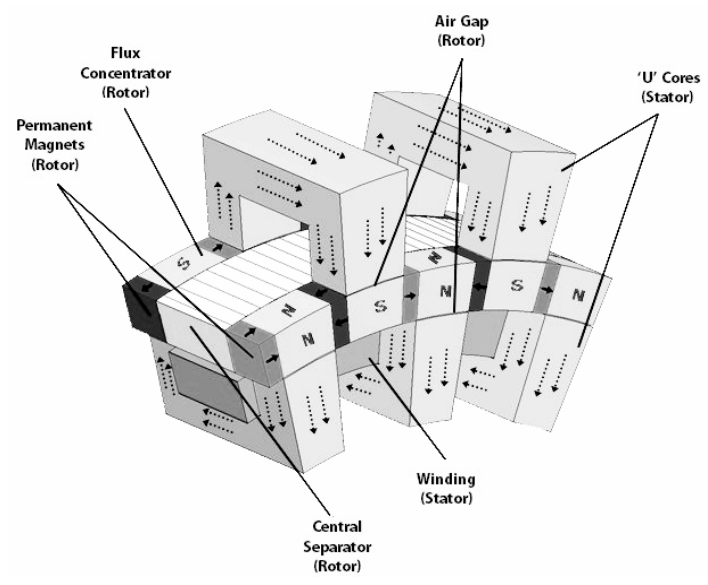

Fig. 1. TFPM with double stator and single winding

The flux concentrator is made of electrical steel due to its high magnetic permeability, in order to aggregate a great amount of magnetic flux, reducing the possible reluctance and leakage flux of the circuit. In this double stator topology poles in ' $U$ ' are displaced in each stator and separated from the rotor by two air gaps for each pole. At the stator, the copper winding is displaced. In this type of topology a double winding may be used. This work makes an initial analysis with one winding displaced at the lower stator due to its simpler and robust construction, as show in Fig. 1. For optimization purposes a deeper analysis with double winding topology is studied later. 


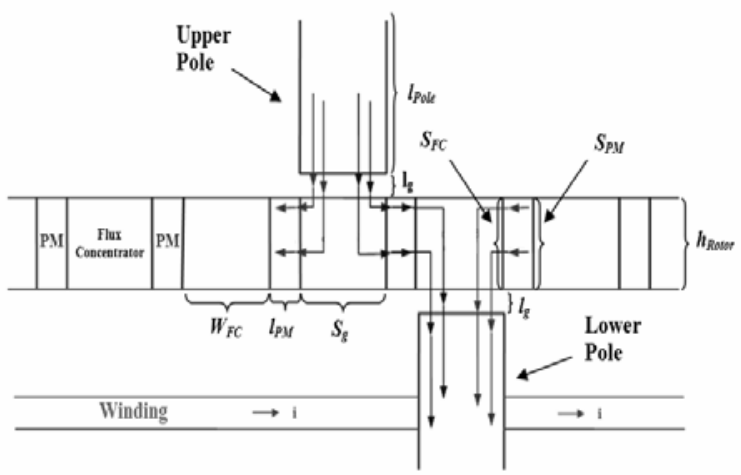

Fig. 2. Magnetic circuit: longitudinal view

Part of the magnetic flux path is presented in Fig. 2, as well as some of the dimensioned machine's parameters. To ease the machine's design and sizing, the following assumptions were made:

- The stator pole length $l_{\text {Pole }}$, is the same for the upper and lower pole;

- The section of both rows of permanent magnets of the rotor's flux concentrators is square and is expressed by $S_{\text {Rotor }}=h_{\text {Rotor }}^{2}$;

- Both permanent magnet and flux concentrator sections have the same value $S_{\mathrm{PM}}=S_{\mathrm{FC}}=S_{\text {Rotor }}$.

\subsection{Working Point}

Reaching a working point that corresponds to an efficient use of the materials and machine dimensions was one of the guidelines of this work.

Through the analysis of the topology's magnetic circuit depicted in Fig. 2, it was observed that the permanent magnet's maximum energy product demands a permanent magnet two times thicker than the air gap distance $\left(l_{\mathrm{PM}}=2 l_{\mathrm{g}}\right)$. This would result in exceedingly long air gaps or extremely thin permanent magnets. To compensate such effect, using a relationship between the permanent magnet and the air gap sections given by a constant $K=S_{\mathrm{g}} / S_{\mathrm{PM}}$, it is possible to achieve an energy product somewhat closer to the material's maximum, allowing adequate air gap and permanent magnet dimensions. From Maxwell's equation that translates Ampere's law, and using Gauss's law for magnetism, the load line expression for the previously described magnetic circuit is given by:

$$
\frac{B_{\mathrm{mp}}}{H_{\mathrm{mp}}}=-K \cdot \frac{\mu_{0} \cdot l_{\mathrm{mp}} \cdot \mu_{\mathrm{fc}} \cdot \mu_{\mathrm{p}}}{\left(h_{\mathrm{rotor}}+w_{\mathrm{fc}}\right) \cdot \mu_{\mathrm{p}}+2 \cdot l_{\mathrm{g}} \cdot \mu_{\mathrm{fc}} \cdot \mu_{\mathrm{p}}+K \cdot l_{\mathrm{p}} \cdot \mu_{\mathrm{fc}}}
$$

Fig. 3 shows the behavior of the maximum value for permanent magnet and ' $U$ ' pole magnetic flux density, depending on the constant $K$. 


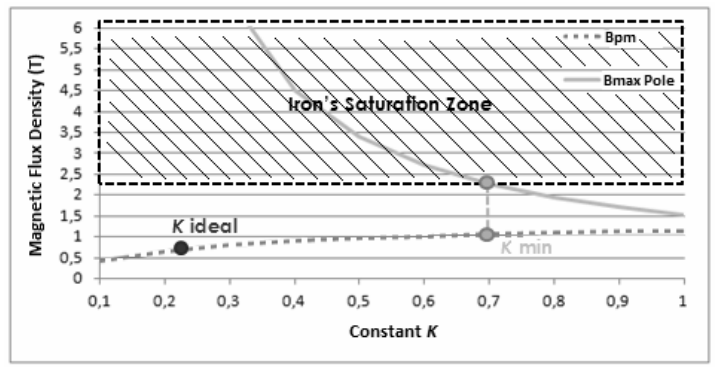

Fig. 3. Magnetic flux density with the variation of $K$

Due to poles shape, and in order to avoid its magnetic saturation, the ideal value for permanent magnets working point could not be reached. Therefore, the next best possible value for $K$ was used. Nevertheless, with some pole shape enhancement, this characteristic can be improved.

For the described sizing a rotor's thickness $h_{\text {Rotor }}$ of $5 \mathrm{~cm}$ and an air gap of $1 \mathrm{~mm}$ were assumed, resulting in a machine's rotor radius of $31 \mathrm{~cm}$.

\subsection{Induced EMF}

In the TFPM topology each flux path is shared by two magnetic circuits as represented in Fig. 2. These circuits can be modeled by the electrical circuit showed in Fig. 4.

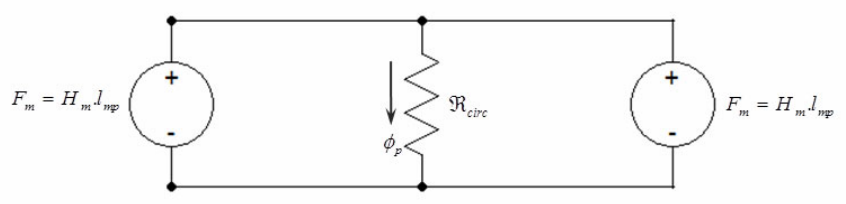

Fig. 4. Representative electrical scheme of one pole pair flux path

As the MMF sources are both equal and in parallel, defining $\mathrm{P}_{\text {Poles }}$ as the number of pole pairs, the induced EMF can be given by:

$$
e(t)=N \cdot P_{P o l e s} \cdot \phi_{P} \cdot \omega \cdot \operatorname{sen}(\omega \cdot t)
$$

\section{Simulations}

In order to verify the analytical expressions concerning machine's sizing and output characteristics, a graphical model of the topology was built and several simulations were performed, through the use of a 3D finite element method (FEM) software. Each of machine's design characteristics was parameterized, allowing an easier analysis of the machine's behavior. 
Fig. 5 shows the magnetic flux density on each point of the constructed model and the flux path along a pole pair, respectively. The registered values matched the working point calculations made at the design phase, with no significant flux leakage observed.

The generator was tested with a purely resistive load sized for a demanding value equal to the nominal electrical power, delivering $8 \mathrm{~kW}$, a value close to the expected. The flux linkage and the respective induced EMF and electrical current curves are shown in Fig. 6.

For each key parameter, various sets of tests were made, varying its value through a specified interval. Fig. 7 (a) depicts the study of the permanent magnet thickness, while maintaining the rotor radius. It was observed that the magnetic saturation is reached for permanent magnets thicker than $8 \mathrm{~mm}$. The chosen value of $1 \mathrm{~cm}$ for the machine's permanent magnet thickness was very close to the optimum value.

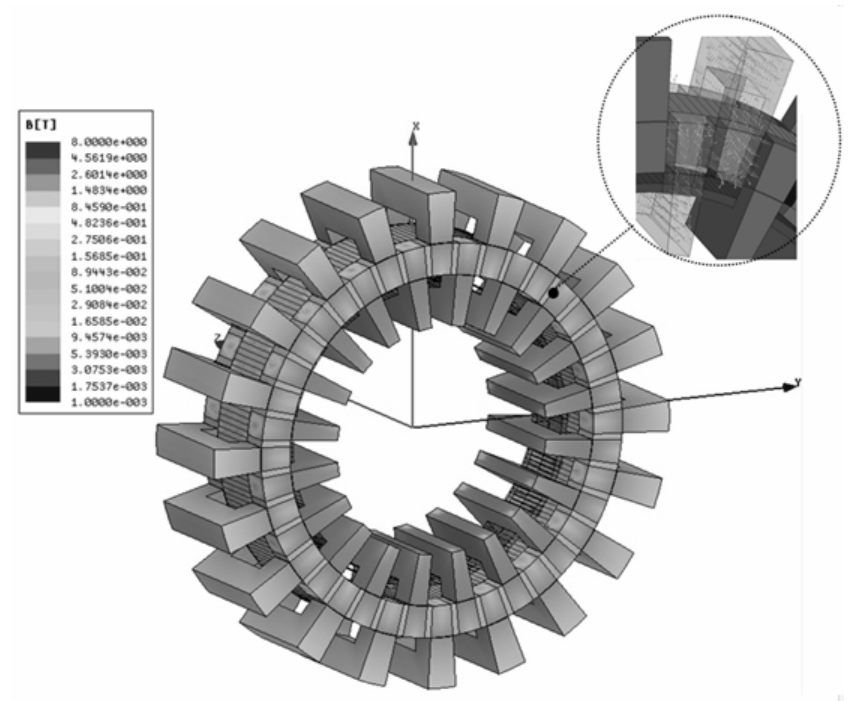

Fig. 5. FEM model's magnetic flux density and flux path along a pole pair
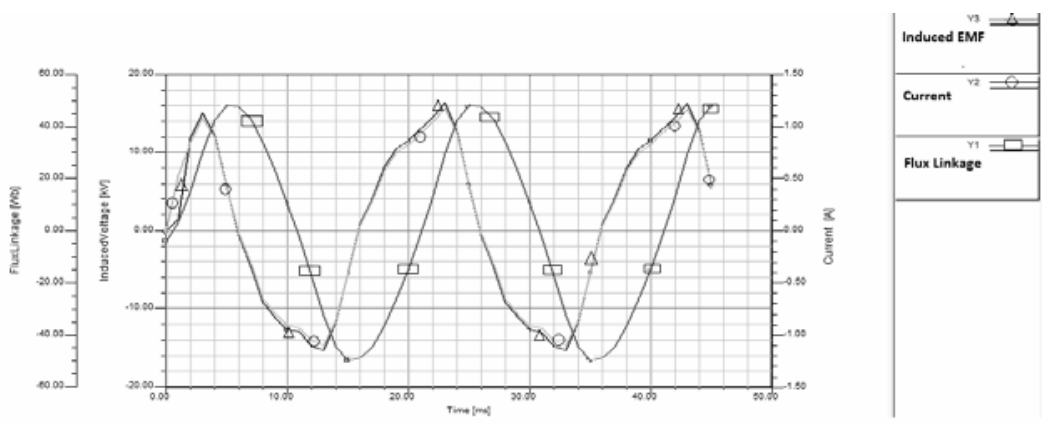

Fig. 6. Flux linkage, induced EMF and electrical current 
For scalability purposes an analysis was made regarding the relationship between the torque density and the overall machine costs through a proportional increment of every machine parameter dimensions, except for the air gap length and the number of winding turns. The calculations were based on the specific costs used in [5]. The plot shown in Fig. 7 (b) reflects an improvement of efficiency in terms of the material's use. This characteristic may be an incentive to apply the TFPM generator in large scale systems.

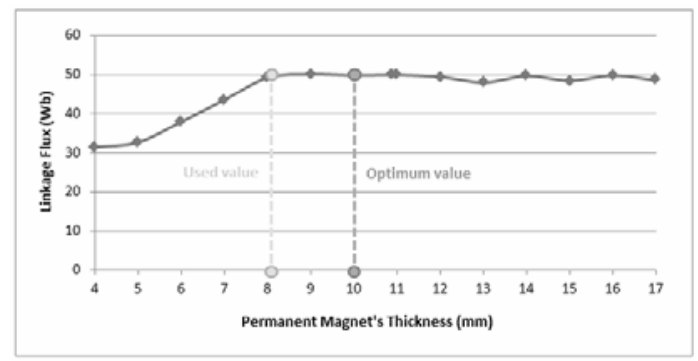

Fig. 7. Permanent magnet's thickness study

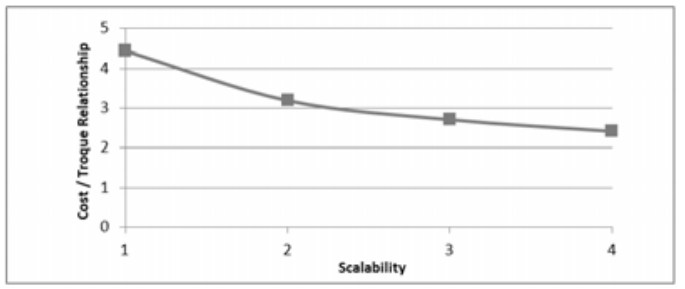

Fig. 8. Study of the scalability effects

\section{Torque Density Optimization}

The generator was sized and tested for a nominal power output of $10 \mathrm{~kW}$, using a 3D FEM software. Although this goal was accomplished, the machine dimensions are underused. The same volume may be used more efficiently, generating more power and therefore improving the machine's torque density. Reinforcing the lower winding with conductors of larger diameter [11] and placing a second winding on the upper stator results in a better use of the permanent magnets material and machine dimensions. Through simulation, it was possible to obtain, with the same volume, an output power of $30,8 \mathrm{~kW}$ and a corresponding value of torque density $\mathrm{T}_{\mathrm{d}}=22,5 \mathrm{kN} \cdot \mathrm{m} \cdot \mathrm{m}^{-3}$, which is close to the values shown in [5].

\section{Conclusions}

The TFPM machine proved to be a good alternative in direct drive ocean wave energy conversion, due to its topology and torque density characteristics, showing results 
consistent with other similar studies and significant advantages over other topologies [5]. The reduction in the Cost/Torque relationship acts an incentive to the use of the TFPM generator in full scale prototypes. As future work is intended a study of the topology under variable speed conditions and an optimization analysis of the ' $U$ ' poles shape for a working point improvement.

\section{References}

1. CEO, Potencial e estratégia de desenvolvimento da energia das ondas em Portugal. Centro de Energia das Ondas (2004)

2. Boud, R.: Status and Research and Development Priorities, Wave and Marine Accessed Energy, Dept. of Trade and Industry (DTI), DTI Report \# FES-R-132, AEAT Report \# AEAT/ENV/1054, United Kingdom (2003)

3. CRES: Ocean Energy Conversion in Europe, Centre for Renewable Energy Sources (2006)

4. Holthuijsen, L.H.: Waves in oceanic and coastal waters. Cambridge University Press, Cambridge (2007) ISBN 0521860288

5. Dubois, M.R., Polinder, H., Ferreira, J.A.: Comparison of generator topologies for directdrive wind turbines. In: Proc. 2000 Nordic Countries Pow. and Indust. Elec., pp. 22-26 (2000)

6. Dubois, M.R.: Optimized Permanent Magnet Generator Topologies for Direct-Drive Wind Turbines. PhD thesis, Delft University of Technology, Delft, The Netherlands (2004)

7. Rang, Y., et al.: Analytical design and modelling of a transverse flux permanent magnet machines. In: IEEE PowerCon 2002, Kunming, China, October 13-17, pp. 2164-2167 (2002)

8. Arshad, W.M., Bäckström, T., Sadarangani, C.: Analytical design and analysis procedure of transverse flux machines. In: IEMDC 2001, pp. 115-121 (2001)

9. Lu, K.Y., Ritchie, E., Rasmussen, P.O., Sandholdt, P.: Modeling and power factor analysis of a single phase surface mounted permanent magnet transverse flux machine. In: Proc. 2003 IEEE Conf. Power Electronics and Drive Systems(PEDS), vol. 2, pp. 1609-1613 (2003)

10. Arnold Magnetic Technologies: Magnets Cathalogs, http: / / www . arnoldmagnetics.com

11. PowerStream: Wire Gauge and Current Limits, http: //www.powerstream.com/Wire_Size.htm 